\title{
Valoración de dietas para alevines de Colossoma macropomum utilizando como fuentes proteicas harinas: de lombriz (Eisenia foetida), soya (Glycine max) y caraotas (Phaseolus vulgaris)
}

\author{
Evaluation of diets for Colossoma macropomum \\ alevins, using earthworm (Eisenia foetida) \\ flour, soybean (Glycine max) and beans \\ (Phaseolus vulgaris) as proteins sources
}

Marielba Morillo S. (1)

Tomas Visbal B. (2)

Daisy Altuve (3)

Fernando Ovalles D. (4)

Ana Luisa Medina G. (3)

\begin{abstract}
The current study was carried out with the purpose of evaluating two alternative diets in the feeding of Colossoma macropomum alevins using earthworm flour (Eisenia foetida), soybean (Glycine max) and beans (Phaseolus vulgaris) as the protein source and as fishmeal substitute and was compared with a control diet. The diets were formulated with a crude protein with a theoretical $32 \%$ protein. The body composition of C. macropomum fed the different diets showed that protein was between $47,3 \%$ and $48,6 \%$ and the lipid content was found between $21,2 \%$ and $24,3 \%$ not showing significant differences between diets $(p>0,05)$. According to the results obtained there is a reason to think that a total substitution of fishmeal by earthworm meal, soybean and beans would be successful in feeding C. macropomum alevins.

Key words: Colossoma macropomum, earthworm flour, soybean, beans, alevins.
\end{abstract}

\section{INTRODUCCIÓN}

La cachama negra (Colossoma macropomum) (Cuvier) es un pez nativo de los ríos Amazonas y Orinoco, cultivado en Colombia, Ecuador, Panamá, Perú, Venezuela y Brasil (1$3)$, con un gran potencial para el cultivo. Este pez de agua cálida requiere de dietas que satisfagan sus requerimientos nutricionales; siendo la proteína uno de los más importantes nutrientes para el rendimiento piscícola, pero a su vez uno de los componentes más costosos de la dieta. Es importante tomar en cuenta el nivel energético de las dietas, ya que niveles muy altos de energía pueden conducir a una disminución del consumo del alimento y niveles muy bajos de energía en la dieta, demanda el uso de la proteína, como fuente energética (4).

Diversos estudios han demostrado que no hay diferencias en la ganancia de peso, cuando la cachama negra fue alimenta-
(1) Postgrado Ciencias Médicas Fundamentales, Facultad de Medicina, ULA, Mérida, Venezuela. (2) Postgrado Química de Medicamentos, Facultad de Farmacia y Bioanálisis, ULA, Mérida, Venezuela.

(3) Departamento de Ciencia de Alimentos-Grupo Ecología y nutrición Facultad de Farmacia y Bioanálisis, ULA, Mérida, Venezuela. (4) Departamento de Análisis y Control, Facultad de Farmacia y Bioanálisis, ULA, Mérida, Venezuela.

Dirigir la correspondencia a:

Marielba Morillo Salcedo Departamento de Ciencias de los Alimentos Facultad de Farmacia y Bioanálisis. ULA Código postal 5101. Mérida- República Bolivariana de Venezuela. Fono Fax: (58)-74-2403473 marimorillo@gmail.com

Este trabajo fue recibido el 9 de Abril de 2013 y aceptado para ser publicado el 22 de Mayo de 2013.

da con dietas isocalóricas (2,7 kcal ED.g-1) y con concentraciones de proteína de 30, 35 y $40 \%$ respectivamente (5-7). Otro ensayo llevado a cabo con dietas (30 \% PB), mostró un mejor resultado cuando tenían un mayor contenido de proteína de origen vegetal (8).

Hay que tomar en cuenta que la mayoría de productos vegetales contienen polisacáridos, que los animales acuáticos no pueden digerir, lo que reduce la eficacia alimentaria, por disminución de la digestibilidad de las grasas y proteínas (9).

La lombriz de tierra (Eisenia foetida), es de gran interés nutricional, ya que es una fuente rica en proteína (> $60 \%$ en base seca), ácidos grasos y minerales (10).

La semilla de soya es considerada una fuente importante de proteína vegetal para la alimentación animal (11), ya que tiene un muy buen perfil de aminoácidos y es rica en vitami- 
nas, para cumplir con los requerimientos nutricionales de los peces $(12,13)$.

El crecimiento de la cachama negra, está determinado por el porcentaje de proteína en la dieta (14), es así que la proteína de la torta de soya se considera un buen sustituto de la proteína de la harina de pescado (15).

Las semillas de leguminosas son el segundo grupo alimenticio en importancia para humanos y animales después de los cereales y son incluso más ricas en proteína que los cereales $(11,16)$.

El Phaseolus vulgaris es una de las leguminosas más consumida, generalmente como granos enteros cocidos, y se le conoce con diversos nombres, en Venezuela como caraotas negras, blancas o rojas según sea su cubierta externa (17).

El objetivo de este trabajo es la valoración de dos dietas alternativas, utilizando como fuente proteica harina de lombriz (Eisenia foetida), soya (Glycine max) y harina de caraotas (Phaseolus vulgaris) y una dieta testigo a base de harina de pescado, en la alimentación de alevines de cachama negra (C. macropomum).

\section{MATERIALES Y MÉTODOS}

La investigación se llevó a cabo en el laboratorio de nutrición acuícola del Departamento de Ciencias de los Alimentos de la Facultad de Farmacia y Bioanálisis, Universidad de Los Andes (ULA), Mérida, Venezuela.

\section{Material biológico}

Los peces fueron criados en la estación piscícola de la UNET en lagunas artificiales, la que suministró 441 especímenes de cachama negra, con un tiempo de vida de 55 días después de la eclosión, se distribuyeron en 9 tanques de $50 \mathrm{~L}$ de capacidad, a razón de 49 peces por cada uno, con un peso promedio de los alevines de 0,85 g $\pm 0,05$. En el manejo de los peces utilizados, se cumplieron las normas éticas exigidas internacionalmente.

Se empleó un sistema de recirculación de agua con flujo continuo $(1,40 \mathrm{l} / \mathrm{min})$ y aireación permanente para conservar los niveles de oxígeno próximos a saturación. El agua fue reciclada utilizando un sistema central con un filtro biológico para remover partículas en suspensión y reducir la concentración de amonio, nitritos y nitratos. Las condiciones se mantuvieron similares al ambiente natural; la temperatura del agua se graduó con un termostato Lifetech Aquarium a $28 \pm 1^{\circ} \mathrm{C}$; el $\mathrm{pH}$ del agua se midió semanalmente y se mantuvo a $7,4 \pm 0,2$; la determinación de nitritos y nitratos se realizó una vez por semana utilizando el kit marca Aquarium Pharmaceuticals (API) y la concentración de los mismos fue $<0,02$ ppm, igualmente se midió el contenido de oxígeno, utilizando un oxímetro Sper Scientific.

La alimentación fue ad libitum alimentándolos tres veces al día, durante 32 días; los peces se pesaron cada 15 días y se determinó el consumo de alimento en ese periodo.

Preparación de las dietas

Se elaboraron 3 dietas experimentales: DSL (harina de soya- harina de lombriz); DSCA (harina de soya -harina de caraotas variedad roja); DT (dieta testigo harina de pescado) (tabla 1).

Las dietas fueron formuladas para obtener un \% teórico de proteína de 32, tomando como referencia a Vásquez-Torres (18); las mezclas de minerales y vitaminas fueron elaboradas cumpliendo con los requisitos de la NRC (12), la harina de lombriz se elaboró en el Departamento de Ciencias de los Alimentos, Facultad de Farmacia y Bioanálisis, ULA, Mérida, Venezuela (10). Se utilizaron insumos de calidad comercial (afrecho, harina de maíz amarillo, caraotas variedad roja y harina de soya) obtenidos en el mercado principal de la localidad. A la harina de soya usada se le extrajo los lípidos con hexano y se secó en estufa MEMMERT a $40^{\circ} \mathrm{C}$, durante 6 horas. Se

\section{TABLA 1}

Insumos, composición porcentual de las tres dietas experimentales

MATERIAS PRIMAS

\begin{tabular}{|c|c|c|c|}
\hline & DSL & DSCA & DT \\
\hline Harina de pescado & 0 & 0 & 37 \\
\hline Harina de soya & 22 & 38 & 0 \\
\hline Harina de caraota variedad rojas & 0 & 34 & 0 \\
\hline Harina de lombriz & 23 & 0 & 0 \\
\hline Harina de maíz amarillo & 12 & 0 & 27 \\
\hline Afrecho de Trigo & 16 & 14 & 8 \\
\hline Almidón gelatinizado & 15 & 0 & 16 \\
\hline Aceite de soya & 6 & 8 & 6 \\
\hline pre mezcla de vitaminas & 1 & 1 & 1 \\
\hline Pre mezcla de minerales & 1 & 1 & 1 \\
\hline Ligantes (CMC) & 1 & 1 & 1 \\
\hline Total & 100 & 100 & 100 \\
\hline
\end{tabular}


empleó carboximetilcelulosa como agente aglutinante no nutritivo. Una vez elaboradas las dietas se guardaron en envases herméticos y refrigeradas a $4^{\circ} \mathrm{C}$.

Para la alimentación de los alevines, la granulometría de las dietas se adaptó a través de tamices a dos tamaños 0,5 $\mathrm{mm}$ y $1 \mathrm{~mm}$, se comenzó a alimentar con gránulos de 0,5 mm. Las dietas se ensayaron por triplicado $(n=3)$.

\section{Análisis químico}

Se realizó el análisis proximal de las tres dietas (DSL, DSCA y DT) y de la carcasa de los peces al inicio y al final del ensayo, para lo cual se determinó el \% de materia seca, proteína, lípidos y cenizas, siguiendo la metodología (19), antes del análisis las muestras se almacenaron en envases herméticos a $4^{\circ} \mathrm{C}$. Los resultados se presentaron en g.100 g-1.

Para la determinación de materia seca se empleó el método de desecación en estufa, para lo cual se utilizó una estufa MEMMERT a $103 \pm 1{ }^{\circ} \mathrm{C}$ durante 24 horas (hasta peso constante), la pérdida de agua en la muestra se calculó por diferencia de peso. El contenido de proteína se obtuvo mediante la cuantificación de nitrógeno total por el método Kjeldahl, utilizando un dispositivo de auto-análisis Kjeltec 2300, después de someter la muestra a digestión en caliente con ácido sulfúrico concentrado en presencia de un catalizador. Para el análisis de lípidos totales se empleó el método soxhlet, para tal fin se empleó el equipo VELP Soxhlet. La determinación del $\%$ cenizas se realizó por incineración de las muestras en mufla Linberg Blue digital a $600^{\circ} \mathrm{C}$, hasta obtener cenizas blancas.

Determinación del perfil de aminoácidos

Se realizó la hidrólisis de las muestras de las tres dietas en estudio, con $\mathrm{HCl} 6 \mathrm{M}$, durante 24 horas a ebullición constante $\left(107-109{ }^{\circ} \mathrm{C}\right)$, previamente se inhibió la oxidación de las mismas utilizando una purga de gas nitrógeno durante dos minutos (20).

La derivatización de las muestras y del estándar (Albumin bovine fraction $V_{1} N^{\circ} 17-8022$ (96-99\%, remanente globulinas) de Sigma Chemical, se Ilevó a cabo empleando el Kit AccQFluor, constituido por AQC diluido en acetonitrilo y disolución tampón de borato sódico 0,2 mM a pH 8.8 (Waters, Milford, MA. EEUU).

El equipo de cromatografía (HPLC) empleado fue el Finnigan Surveyor Plus de Thermo-fisher, que incluye: bomba cuaternaria con desgasificador incorporado, muestreador de múltiple capacidad automático, inyector automático de toma variable $(5-25 \mu \mathrm{l})$. Un horno integrado al automuestreador, un calefactor integrado con el porta columna, un detector de fluorescencia. Sistema de adquisición y procesado de datos mediante el software ChromQuest, versión 4.2 (de la misma marca).

Se usó la columna Nova-Pak C18, $4 \mu \mathrm{m} ; 3,9$ × $150 \mathrm{~mm}$ (Waters). Milford, MA, EE.UU). La detección se llevó a cabo por fluorescencia (excitación: 250 nm y emisión: 395 nm). El volumen de inyección fue $10 \mu \mathrm{l}$. Se empleó un gradiente ternario modificado y adaptado a la respuesta del sistema cromatográfico.

\section{Parámetros zootécnicos y retenciones}

Se realizaron controles zootécnicos cada 15 días, para lo cual se determinaron: peso inicial y final de los peces, consumo total de alimentos y mortalidad, estos datos permitieron calcular los siguientes parámetros (21).

- Ganancia de peso total: GPT= Peso corporal final (PCF) -Peso corporal inicial (PCI)

- Ganancia de peso en \% de peso inicial: GP\%PI=GPT x100/ ( $\mathrm{PCl} \times($ dias -1$)$

- I Índice de consumo: IC= (Consumo.\%MS) /GPT

- Tasa de crecimiento especifica: TCE=log (Peso medio final)- $\log$ (Peso medio inicial) $\times 100 /$ (días-1)

- Eficiencia alimenticia: $E A=$ Peso ganado $(g) / a l i m e n t o$ ingerido ( $g$ ).

- Índice de crecimiento diario: $I C D=100 \times((P C F) 1 / 3-$ (PCI)1/3)/duración (días))

- Coeficiente de eficiencia proteica: $C E P=$ GPT/proteína cruda ingerida

- $\quad$ Peso corporal medio: $\mathrm{PCM}=(\mathrm{PCl}+\mathrm{PCF}) / 2$

- Retención: $\mathrm{R}=((\mathrm{PCF} \times$ composición final del pez $)-(\mathrm{PCI} \times$ composición inicial del pez))/ (CONS $x$ composición del alimento) $\times 100$

- Consumo (día): CONS=g de alimento por tanque/ $\mathrm{N}^{\circ}$ de peces (22).

- $\%$ Sobrevivencia: \% SOBREV $=100 \times\left(\left(\mathrm{N}^{\circ}\right.\right.$ inicial $-\mathrm{N}^{\circ}$ final) $/ \mathrm{N}^{\circ}$ inicial)) (22).

\section{Análisis estadístico}

Se realizó un estudio estadístico de los resultados con el programa Startgraphic Centurion $X V$, se realizó análisis de varianza ANOVA simple con test Student de Newman Keuls.

TABLA 2

Composición química de las dietas $(X \pm D E)$

\begin{tabular}{|c|c|c|c|}
\hline \multirow[t]{2}{*}{ Composición química } & \multicolumn{3}{|c|}{ Dietas } \\
\hline & DSL & DSCA & DT \\
\hline MS (g.100 g $\left.\mathrm{g}^{-1}\right)$ & $89,1 \pm 0,6^{b}$ & $90,5 \pm 0,1^{\mathrm{a}}$ & $88,5 \pm 0,1^{a, c}$ \\
\hline Proteína cruda (g.100 $\left.\mathrm{g}^{-1} \mathrm{MS}\right)$ & $33,4 \pm 0,3$ & $31,6 \pm 0,1$ & $31,8 \pm 0,2$ \\
\hline Lípido crudo (g.100 g-1MS) & $8,8 \pm 0,1^{a, b}$ & $7,7 \pm 0,1^{b}$ & $9,5 \pm 0,1^{a}$ \\
\hline Cenizas (g.100 $\left.\mathrm{g}^{-1} \mathrm{MS}\right)$ & $6,1 \pm 0,01^{\mathrm{a}}$ & $5,7 \pm 0,1^{b}$ & $8,6 \pm 0,2^{a}$ \\
\hline${ }^{1}$ Energia digestible (cal.100 g-1 $)$ & 351,6 & 374,8 & 393,2 \\
\hline${ }^{2}$ Relacion ED/PB & 10,5 & 11,9 & 12,3 \\
\hline \multicolumn{4}{|c|}{$\begin{array}{l}\text { Letras diferentes en los valores indican diferencias significativas }(p<0,05) \\
\text { Dietas: DSL (h. soya: } h \text {. lombriz); DSCA (h. soya: } h \text {. caraota); DT (h. pescado) } \\
\text { ED calculada tomando como base los valores fisiológicos estándar de energía digestible para peces: proteínas 4,54, lípidos 8,6 y carbohidratos 3,58 (23). } \\
\text { Relación teórica estimada a partir de niveles de Energía Digestible (ED) calculada y Proteína Bruta (PB) formulada }\end{array}$} \\
\hline
\end{tabular}




\section{RESULTADOS}

Composición química de las dietas: La composición química de las dietas utilizadas para alimentar los alevines de cachama negra se presentan en la tabla 2 , se observaron diferencias significativas entre datos $(p<0,05)$.

Perfil de aminoácidos de las dietas: En la tabla 3, se presentan los resultados del contenido de aminoácidos en las tres dietas y como referencia: torta de soya, frijol (P. vulgaris), harina de lombriz y las necesidades de aminoácidos para juveniles de cachama blanca (P. brachipomus).

Parámetros de crecimiento

En la tabla 4, se presentan los resultados del crecimiento de los alevines de cachama negra, encontrando diferencias significativas entre datos en función del alimento recibido $(p<0,05)$.

Composición corporal de los peces

La composición química de la carcasa de los peces, expresados en base seca, se presentan en la tabla 5, determinada al inicio del ensayo sobre una muestra de peces representativa (inicial) y al final del experimento en la totalidad de peces por tanque por cada tratamiento (19). Sólo el \% de cenizas, mostró diferencias significativas $(p<0,05)$, los demás datos no presentaron diferencias significativas $(p>0,05)$.
Retenciones de proteína y lípidos en alevines de cachama negra, alimentados con dietas experimentales durante 32 días

Los resultados de retenciones de proteínas, lípidos y nitrógeno ganado en alevines de cachama negra, alimentados con diferentes dietas experimentales se presentan en la tabla 6 . Los resultados de retención de lípidos no fueron significativamente diferentes $(p>0,05)$, mientras que las retenciones de proteínas sí lo fueron $(p<0,05)$.

\section{DISCUSIÓN}

La valorización de fuentes proteicas como las harinas de lombriz, soya y caraotas para la alimentación de alevines de cachama negra (C. macropomum), es de importancia dado que no hay reportes donde se haya sustituido la harina de pescado, con mezclas de estas materias primas. Estos resultados podrían tener repercusión en la dependencia de fuentes proteicas importadas.

Se observó de manera directa (visual), que los peces presentaron una buena aceptación del alimento y gran voracidad. El \% de sobrevivencia estuvo entre 93,2 y 98,4\% (tabla 4), no observando mortalidad relacionada con la composición de las dietas.

La energía digestible teórica (ED) de las dietas (tabla 2) se encontró entre 351,6 y 393,2 (cal.100 g-1), presentando la

TABLA 3

Contenido de aminoácidos proteicos $(X \pm D E)$, en la dietas utilizadas para alimentar los alevines de C. macropomum

\begin{tabular}{|c|c|c|c|c|c|c|c|}
\hline \multirow[b]{3}{*}{ Aminoácidos } & \multicolumn{7}{|c|}{ Composición de aminoácidos g.kg-1 } \\
\hline & \multicolumn{3}{|c|}{ Dietas } & \multicolumn{3}{|c|}{ Referencias } & \multirow[b]{2}{*}{$\begin{array}{l}{ }^{4} \text { Requerimientos } \\
\text { para juveniles de } \\
\text { cachama blanca }\end{array}$} \\
\hline & DSL & DSCA & DT & $\begin{array}{l}{ }^{1} \text { Torta } \\
\text { de soya }\end{array}$ & $\begin{array}{c}{ }^{2} \text { Frijol } \\
\text { (P. vulgaris) }\end{array}$ & $\begin{array}{l}{ }^{3} \text { Harina de } \\
\text { lombriz } \\
\text { (E. foetida) }\end{array}$ & \\
\hline${ }^{*}$ Arginina & $21,7 \pm 0,1$ & $26,5 \pm 0,1$ & $37,1 \pm 0,5$ & 75,7 & 12,3 & - & 16,2 \\
\hline${ }^{*}$ Histidina & $9,3 \pm 0,8$ & $10,6 \pm 0,1$ & $17,5 \pm 0,6$ & 26,5 & 6,3 & 25 & 5,6 \\
\hline${ }^{*}$ Isoleucina & $30,1 \pm 0,1$ & $24,5 \pm 0,1$ & $29,6 \pm 0,1$ & 45,3 & 9,3 & 62 & 10,5 \\
\hline${ }^{*}$ Leucina & $47,6 \pm 0,2$ & $37,4 \pm 0,2$ & $48,6 \pm 0,2$ & 77,9 & 16,8 & 166 & 12,4 \\
\hline *Lisina & $50,1 \pm 0,1$ & $28,7 \pm 0,1$ & $47,7 \pm 0,7$ & 65,1 & 15,9 & 43 & 18,1 \\
\hline${ }^{*}$ Metionina & $5,7 \pm 0,1$ & $5,4 \pm 0,3$ & $16,4 \pm 0,1$ & 12,7 & 2,4 & - & 6,2 \\
\hline *Fenilalanina & $24,3 \pm 0,1$ & $22,7 \pm 0,2$ & $26,3 \pm 0,6$ & 49,5 & 11,5 & 35 & 10,3 \\
\hline *Triptófano & nd & nd & nd & 14,2 & - & - & - \\
\hline *Treonina & $9,1 \pm 0,2$ & $7,8 \pm 0,1$ & $24,1 \pm 0,8$ & 39,7 & 8,8 & 36 & 11,2 \\
\hline *Valina & $19,5 \pm 0,1$ & $25,2 \pm 0,1$ & $32,04 \pm 0,1$ & 45,0 & 10,2 & - & 12,4 \\
\hline${ }^{* *}$ Tirosina & $17,2 \pm 0,1$ & $24,4 \pm 0,2$ & $24,3 \pm 0,3$ & 35,0 & 5,6 & 9 & - \\
\hline Ácido aspártico & - & $44,9 \pm 0,1$ & $48,7 \pm 3,4$ & - & - & 71 & - \\
\hline Serina & - & $4,6 \pm 0,1$ & $22,6 \pm 0,1$ & - & - & 9 & - \\
\hline Acido glutámico & $53,5 \pm 4,1$ & $74,9 \pm 0,5$ & $95,3 \pm 0,6$ & - & - & 90 & - \\
\hline Glicina & $18,0 \pm 1,5$ & $19,6 \pm 0,1$ & $34,2 \pm 0,2$ & - & - & 57 & - \\
\hline Alanina & $16,3 \pm 0,2$ & $17,8 \pm 0,03$ & $35,9 \pm 0,6$ & - & - & - & - \\
\hline \multicolumn{8}{|c|}{ 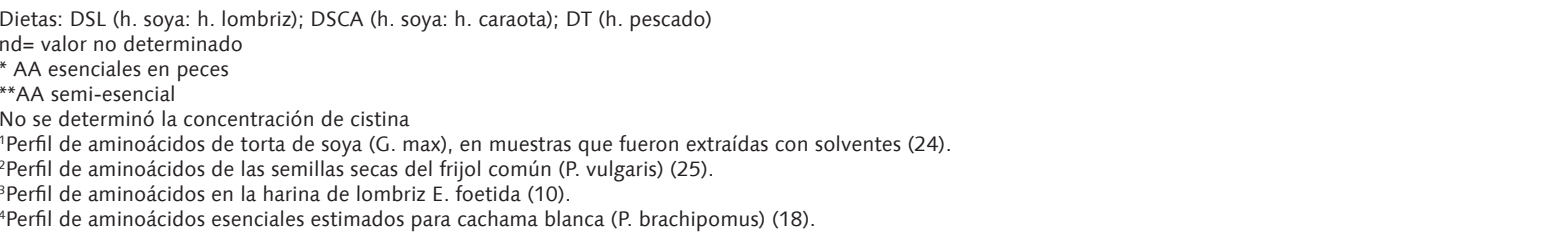 } \\
\hline
\end{tabular}


dieta DT un valor superior, se puede suponer que altos niveles de energía en la dieta pueden reducir el consumo total de alimento (26), podríamos presumir que es por esta razón, que la dieta DT que presenta una ED mayor, mostró un consumo menor (tabla 4).

La calidad de la proteína utilizada para la formulación de dietas para peces depende de la composición de aminoácidos y de su disponibilidad biológica, es decir entre más se aproxime el contenido de aminoácidos esenciales a los requerimientos de la especie a estudiar, mayor será su valor nutricional $(24,27)$.

Es indispensable la presencia de diez aminoácidos esenciales en la dieta de los peces: arginina, lisina, histidina, isoleucina, leucina, metionina, fenilalanina, treonina, triptófano y valina. Los requerimientos nutricionales de estos aminoácidos no son conocidos para todas las especies de peces, sin embargo existen datos para las especies más estudiadas $(12,28)$.

Según Vásquez-Torres (18) el requerimiento de lisina para juveniles de cachama blanca es 18,1 g.kg-1, mientras que para el pacú (P. mesopotamicus) que es considerado un aminoácido indispensable, es 17,7 g.kg-1 $(29,30)$. En las dietas (DSL, DSCA y DT) este aminoácido se encuentra entre 28,7 y 50,1 g.kg-1 (tabla 3).

Otro aminoácido necesario para el crecimiento del pez, es la arginina, en las dietas estos valores se encuentran entre 21,7 y 37,1 g.kg-1 y el requerimiento para juveniles de cachama blanca, es de 16,2 g.kg-1(18).

Asimismo la histidina, es un aminoácido que interviene en la formación de la estructura terciaria de las proteínas, en nuestras dietas se encontró entre 9,3 y 17,5 g.kg-1 superior al requerimiento para juveniles de cachama blanca que es de 5,6 g.kg-1(18) y de juveniles de pacú de 12,4 g.kg-1 (30).

Cabe señalar que las dietas tienen un alto contenido de estos tres aminoácidos, por lo que deberían cubrir la necesidad nutricional de los alevines de C. macropomum.

Los demás aminoácidos esenciales (tabla 3), cumplen con los requerimientos para juveniles de cachama blanca (18),

TABLA 4

Resultados de crecimiento $(X \pm D E)$ de los alevines de cachama negra (C. macropomum) alimentados con dietas experimentales durante 32 días.

\begin{tabular}{lccc} 
Datos zootécnicos & & DIETAS & \\
& DSL & $0,87 \pm 0,1 \mathrm{a}$ & DT \\
PMI & $0,82 \pm 0,02 \mathrm{a}$ & $9,9 \pm 0,1 \mathrm{a}$ & $0,92 \pm 0,1 \mathrm{~b}$ \\
PMF & $8,9 \pm 1,3 \mathrm{a}$ & $220,8 \pm 24,5$ & $216,8 \pm 15,5$ \\
GPT & $266,1 \pm 43,5$ & $971,2 \pm 73,1 \mathrm{a}$ & $599,6 \pm 47,1 \mathrm{~b}$ \\
GP\%PI & $990,1 \pm 192,9 \mathrm{a}$ & $1,22 \pm 0,01$ & $1,11 \pm 0,1$ \\
IC & $1,04 \pm 0,1$ & $0,82 \pm 0,01$ & $0,90 \pm 0,1$ \\
F Eff & $0,98 \pm 0,1$ & $2,42 \pm 0,02 \mathrm{a}$ & $2,84 \pm 0,2 \mathrm{ab}$ \\
CEP (PER) & $3,08 \pm 0,4 \mathrm{~b}$ & $7,40 \pm 0,2 \mathrm{a}$ & $6,07 \pm 0,2 \mathrm{~b}$ \\
TCE (\%) & $7,43 \pm 0,6 \mathrm{a}$ & $3,6 \pm 0,1 \mathrm{a}$ & $2,8 \pm 0,1 \mathrm{~b}$ \\
ICD (\%) & $3,5 \pm 0,4 \mathrm{a}$ & $302,1 \pm 18,1$ & $270,9 \pm 3,4$ \\
CONS (g) & $300,5 \pm 8,5$ & $97,1 \pm 1,2 \mathrm{~b}$ & $98,4 \pm 0,3 \mathrm{c}$ \\
\% SBRV & $93,2 \pm 0,1 \mathrm{a}$ & & \\
\hline
\end{tabular}

Letras diferentes en los valores indican diferencias significativas ( $p<0,05)$, algunos resultados no son significativamente diferentes ( $p>0,05)$.

Dietas: DSL (h. soya: h. lombriz); DSCA (h. soya: h. caraota); DT (h. pescado)

PMI: Peso medio inicial; PMF: Peso medio final; GPT: Ganancia de peso total; GP\%PI: Ganancia de peso en porcentaje de peso inicial; IC: Índice de consumo;

Feff: Eficiencia alimenticia; CEP: Coeficiente de eficacia proteica; TCE: Tasa de crecimiento especifico; ICD: Índice de crecimiento diario;

CONS: Consumo; \% SBRV= \% de sobrevivencia.

\section{TABLA 5}

Composición corporal $(X \pm D E)$ de los alevines de cachama negra (C. macropomum), alimentados con dietas experimentales durante 32 días.

Inicial

$\begin{array}{lll}\text { Matéria seca (\%) } & 96,3 \pm 0,1 & 95,8 \pm 1,5 \\ \text { Proteína (\%MS) } & 57,0 \pm 0,1 & 47,3 \pm 0,9 \\ \text { Lípidos (\% MS) } & 25,5 \pm 0,3 & 24,3 \pm 1,1 \\ \text { Cenizas (\% MS) } & 10,4 \pm 0,2 & 9,3 \pm 0,4^{\mathrm{a}}\end{array}$

Dietas

DSCA

DT

$\begin{array}{ll}93,9 \pm 0,6 & 93,8 \pm 0,8 \\ 48,6 \pm 0,8 & 47,7 \pm 0,7 \\ 21,2 \pm 1,2 & 23,9 \pm 1,0 \\ 10,0 \pm 0,2^{\mathrm{a}} & 12,2 \pm 0,5^{\mathrm{b}}\end{array}$

Los datos se presentan corregidos por \% de materia seca. Letras diferentes indican que existen diferencias significativas $(p<0,05)$.

Dietas: DSL (h. soya: h. lombriz); DSCA (h. soya: h. caraota); DT (h. pescado). 
con excepción de la metionina que en las dietas DSL y DSCA se encuentra entre 5,4 y 5,7 g.kg-1, inferior al requerimiento $(6,2$ g.kg-1) (18).

El criterio más sencillo para evaluar el crecimiento del pez, es la ganancia de peso total (GPT), sin embargo los indicadores más utilizados para determinar cuándo una dieta es mejor que otra, en función del contenido de nutrientes son: la tasa de crecimiento específico (TCE), ganancia de peso por porcentaje de peso inicial (GP\%PI) y el coeficiente de eficiencia proteica (CEP). Para medir la eficacia de las dietas, se utilizó el índice de consumo (IC).

Los resultados de ganancia de peso total (GPT) (tabla 4), indican que en los alevines de cachama negra, alimentados con las dietas DSL, DSCA y DT, no se observó variación del crecimiento a pesar de que las fuentes proteicas eran diferentes.

Estudios reportados por Vásquez-Torres (31), quien probó dietas entre 16 y 36 (\%PB) en juveniles de cachama blanca, mostraron que el crecimiento aumentó proporcionalmente con la concentración proteica hasta 32\%; por encima de este nivel el crecimiento disminuyó significativamente.

Pezzato et al. (32), evaluaron dietas de 3 niveles proteicos 24, 28 y 32 \% PB en alevines de Piauçu (Leporinus macrocephalus) y observaron el mejor crecimiento con $28 \%$ PB y 2,8 Kcal ED. g PB -1.

En cuanto a la TCE (tabla 4), los mejores resultados se observaron en los peces alimentados con las dietas DSL y DSCA, que mostraron TCE superiores a los peces alimentados con DT.

Los valores de TCE obtenidos en esta investigación, fueron superiores a los suministrados por Van der Mer et al. (33) que alimentó alevines de cachama negra, utilizando dietas a base de soya (35\% PB) y harina de pescado (31,5\% PB), mostrando una TCE de 4,5\%, para la dieta a base de harina de pescado y $3,9 \%$ con la dieta a base de harina de soya.

La GP\%PI de los peces alimentados con DSL y DSA resultaron superiores a la de los peces alimentados con la dieta DT (tabla 4).

Vásquez-Torres et al. (31) reportaron valores de $270 \%$ en juveniles de cachama blanca alimentados con una dieta 57,8 \% PB. En nuestra experiencia la GP\%PI fue superior, con dietas con un \% PB inferior. Esto indica que hubo un crecimiento rápido de los alevines durante el tiempo que se alimentaron con las dietas en estudio, lo que nos permite inferir el buen aprovechamiento por parte del pez de los nutrientes en las dietas.

Para caracterizar la utilización de proteínas se recurrió a criterios como el CEP, que se corresponde a la cantidad de proteína de la dieta que fue convertida en peso corporal. En nuestra investigación el CEP de las dietas DSCA y DSL mostraron diferencias $(p<0,05)$ pero no se observaron diferencias cuando se compararon con DT $(p>0,05)$ (tabla 4). Nuestros resultados son similares a los reportados por Vásquez-Torres et al. (31), en juveniles de cachama blanca, alimentados con dieta (32,3\% PB), que mostraron CEP de 2,39.

En cuanto a la eficacia de las dietas, el IC es uno de los parámetros más utilizado, que relaciona el consumo de la dieta con la ganancia de peso del pez. Los valores de IC no mostraron diferencias $(p>0,05)$. Indicando que hubo buena conversión del alimento por parte del pez, sin observarse que haya ocurrido pérdida del alimento durante el suministro de las dietas a los peces (tabla 4).

En la tabla 5 se presenta la composición proximal de la carcasa de los peces (19), el valor en porcentaje de proteína (PB), se encontró entre 47,3 y 48,6 y el contenido de lípidos entre 21,2 y $23,9 \%$, no se observaron diferencias $(p>0,05)$. Los valores de \% PB y \% de lípidos, difieren de los reportados por Morillo et al. (34), en alevines de cachama negra, alimentados con dietas a base de Erythrina edulis y soya (28,9 a 32,5\%PB), quienes obtuvieron valores superiores de $\% \mathrm{~PB}(66,03$ y 66,81$)$ y valores inferiores de contenido de lípidos (11,26 a 16,03\%).

Podríamos suponer que a pesar de que en este ensayo la TCE fue elevada, no se observó que el aprovechamiento de la proteína para la formación de músculo haya sido óptimo, posiblemente la presencia de un alto contenido de carbohidratos en las dietas haya interferido de alguna forma.

Los resultados de retenciones de proteínas, lípidos y $\mathrm{N}$ ganado en alevines de cachama negra, están en la tabla 6. Los resultados de retención de proteínas son significativamente diferentes $(p<0,05)$, mientras que los resultados de retención de lípidos no presentan diferencias $(p>0,05)$.

Se concluye que es viable, utilizar la mezcla de harina de lombriz y harina de soya, o harina de soya y harina de caraotas, para sustituir la harina de pescado en las dietas para alevines de cachama negra (C. macropomum). Sería conveniente realizar otras pruebas sobre el pez que permitan determinar si existen daños esqueléticos o toxicidad hepática causados por algunos de los componentes de la dieta.

\section{RESUMEN}

El presente estudio se realizó con el objetivo de valorar dos dietas alternativas en la alimentación de alevines de

\section{TABLA 6}

Retenciones de proteínas y lípidos $(X \pm D E)$, en alevines de cachama negra (C. macropomum), alimentados con dietas experimentales durante 32 días.

DSL

DSCA

DT

Retenciones (\% ingerido)

Materia seca (MS)

Proteinas (\%)

$47,7 \pm 4,5^{\mathrm{a}}$

$78,2 \pm 1,6^{a}$

Lípidos(\%)

$215,5 \pm 42,4$

$\mathrm{N}$ ganado (mg/ kg ABW/d)

Grasa ganada (g/kg ABW/d)
$3052,5 \pm 19,4^{a}$

$5,72 \pm 1,4$
$87,2 \pm 12,3^{b}$

$133,4 \pm 23,4^{b}$

$222,3 \pm 27,6$

$3634,6 \pm 221,8^{b}$

$5,69 \pm 1,2$
$83,5 \pm 4,2^{b}$

$138,6 \pm 1,6^{b}$

$205,5 \pm 27,2$

$3403,9 \pm 61,3^{b}$

$6,40 \pm 0,6$

Letras diferentes indican diferencias significativas $(p<0,05)$

Dietas: DSL (h. soya: h. lombriz); DSCA (h. soya: h. caraota); DT (h. pescado) 
cachama negra (Colossoma macropomum), utilizando como fuentes proteicas harinas de lombriz (Eisenia foetida), soya (Glycine max) y caraotas (Phaseolus vulgaris) como sustituto de la harina de pescado, comparándolo con una dieta testigo. Las dietas se formularon con un porcentaje teórico de proteína bruta de $32 \%$. En cuanto a la composición corporal de las cachamas alimentadas con las dietas, el \% de proteína se determinó entre 47,3 y 48,6 y \% de lípidos entre 21,2 y 24,3; sin presentar diferencias significativas $(p>0,05)$. De acuerdo a los resultados se puede concluir que una sustitución total de la harina de pescado por harina de soya- lombriz, y de soyacaraota conduciría a buenos resultados para la alimentación de alevines de cachama negra.

Palabras clave: Colossoma macropomum; harina de lombriz; soya; caraotas; alevines.

Agradecimientos: Este trabajo contó con el apoyo de los proyectos FONACIT: G2005000869 y G2008001102 ; de los proyecto CDCH-TA: FA-434-08-03-ED, FA-492-11-08-A y FA-511-12-08-B; del Departamento de Ciencia de Alimentos de la Facultad de Farmacia y Bioanálisis, ULA, del laboratorio de Inmuno Diagnóstico, del Instituto de Inmunología Clínica (IDIC), IAHULA; de la Estación piscícola de la Universidad del Táchira (UNET), Venezuela.

\section{BIBLIOGRAFÍA}

1. Soares LH. Cultivo experimental de tambaqui, Colossoma macropomum Cuvier, 1818 usando cuatro tipos de dietas. In: Chellappa S, Chellappa NT, Barbosa WB, Huntingford FA, Beveridge MCM. Growth and production of the Amazonian tambaqui in fixed cages under different feeding regimes, Aquacult Int. 1995; 3:11-21.

2. Armando HR. Cultivo de Colossoma. In: Chellappa S, Chellappa NT, Barbosa WB, Huntingford FA, Beveridge MCM. Growth and production of the Amazonian tambaqui in fixed cages under different feeding regimes. Aquacult Int. 1995; 3:11-21.

3. Barbosa WG. Cultivo experimental de larvas de tambaqui, Colossoma macropomum em condicoes experimentais, utilizando duas dietas. In: Chellappa S, Chellappa NT, Barbosa WB, Huntingford FA, Beveridge MCM. Growth and production of the Amazonian tambaqui in fixed cages under different feeding regimes, Aquacult Int. 1995; 3:11-21.

4. Gutierrez AFW, Zaldivar RJ, Contreras SG. Efectos de varios niveles de energía digestible y de proteína en la dieta sobre el crecimiento de Gamitana Colossoma macropomum (cachama) CUVIER 1818, Rev Inv Vet Perú 2009; 20:178-86.

5. Merola N. Cantelmo OA. Growth, feed convertion, and mortality of cage reared "tambaquí", Colossoma macropomum, fed various dietary feeding regimes and protein Piaractus brachypomus levels, Aquaculture 1987; 66:223-33.

6. Macedo-Viegas EM. Necessidade proteica na nutricao do tambaqui Colossoma macropomum Cuvier 1818 (Pisces Characidae). Thesis M Sc. Univ. Estadual Paulista, Jabocatibal, SP. 1979.

7. Carniero DJ. Digestibilidade proteica em dietas isocalóricas para o tambaquí Colossoma macropomum (Cuvier, Pisces), Ann. $2^{\circ}$ Simp Bras Aquicult. E $2^{\circ}$ Ene. Nac Ranicult Brasilia, 1981, p 788-800.

8. Werder UU. Saint Paul U. Feeding trials with herbivorous and omnivorous. Amazonian fishes. Aquaculture 1978; 15:175-7.

9. Médale F. Kaushik S. Les sources protéiques dans les aliments pour les poissons d'élevage, Cah Agric 2009; 18:103-11.

10. Vielma RA. Estudio Físico- Químico y Funcional de las Proteínas de la Lombriz Eisenia foetida. Tesis de Doctorado. ULA. Mérida, Venezuela. 2004.

11. Bressani R. Elias LG. Nutritional value of legume crops for humans and animals. In: Summerfield RJ. Bunting AH. (Eds) Advances in Legume Science Royal Botanic Gardens, London, U. K. 1980. p 135-55.

12. National Research Council (NRC). Nutrient requirements of warm water fishes and shellfishes. National Academy Press, Washington, D.C., 1993.

13. Lim C, Klesius PH, Higgs DA. Substitution of canola-meal for soybean-meal in diets for channel catfish Ictalurus punctatus, J World Aquac Soc. 1998; 29:161-8.

14. Van der Meer MB, Machiels MAM, Verdegem MCJ. The effect of dietary protein level on growth protein utilization and body composition of Colossoma macropomum (Cuvier), Aquacult Res. 1995; 26:901-9.

15. Van der Meer MB, Verdegem MCJ. Comparison of amino acid profiles of feeds and fish as a quick method for selection of feed ingredients: a case study for Colossoma macropomum (Cuvier), Aquacult Res 1996; 27:487-95.

16. Todorov NA. Cereals pulses and oilseeds, Livest Prod Sci. 1988; 19:47-95.

17. Sangronis $E$, Machado C, Cava R. Propiedades funcionales de las harinas de leguminosas (Phaseolus vulgaris y Cajan cajan) germinadas. Interciencia 2004; 29:80-5.

18. Vásquez-Torres W. Determinação das exigencias de proteinas, gordura e carboidratos em dietas para crescimento de juvenis de pirapitinga. Piaractus brachypomus (CUVIER 1818). In: Vásquez-Torres W. Principios de nutrición aplicada al cultivo de peces. Juan XXIII. Instituto de Acuicultura Universidad de Los Llanos. Colombia. 2004.

19. Association of Official Analytical Chemists. Official Methods of Analysis of the AOAC. 17th ed. Gaithersburg M. D, USA, 2000.

20. Cohen SA. Michaud DP. Synthesis of a fluorescent derivatizing reagent, 6-aminoquinolyl-N-hydroxysuccinimidylcarbamate, and its application for the analysis of hydrolysate amino acids via high-performance liquid chromatography, Anal. Biochem, 1993; 211:279-87.

21. Guillaume J, Kaushik S, Bergot P, Metailler R. Nutrition et alimentation des poisons et crustacés, INRA/INFREMER. París, France. 1999.

22. López OYM, Vásquez TW, Wills FA. Evaluación de diferentes proporciones de energía/ proteína en dietas para juveniles de yamú Brycon siebenthalae (Eigenmann, 1912), Orinoquia. 2004; 8:64-76.

23. De Silva SS. Anderson TA. Fish Nutrition in Aquaculture. Chapman \& Hall. London, UK. 1995.

24. Vásquez-Torres W. Principios de nutrición aplicada al cultivo de peces. Juan XXIII. Instituto de acuicultura. Universidad de Los Llanos. Colombia. 2004.

25. Tacon AG. Nutrición y alimentación de peces y camarones cultivados. FAO-ITALIA. 1999.

26. Gutiérrez FW, Quispe M, Valenzuela L, Contreras $G$, Zaldívar J. Utilización de la proteína dietaría por alevinos de la gamitana, Colossoma macropomum, alimentados con dietas isocalóricas, Rev Peru Biol. 2010; 17:219-23.

27. Berge GE, Sveier $H$, Lied E. Effects of feeding Atlantic salmon (Salmo salar L) imbalanced levels of lysine and arginine, Aquacult Nutr. 2002; 8:239-48.

28. Tibaldi E. Kaushik SJ. Amino-acid requirements of mediter- 
ranean fish species. Cah Options Mediterr. 2005; 63:59-65.

29. Abimorad EG, Favero GC, Squassoni GH, Carneiro D. Dietary digestible lysine requirement and essential amino acid to lysine ratio for pacú Piaractus mesopotamicus, Aquacult Nutr. 2010; 16:370-7.

30. Dabrowski K, Arslan M, Terjesen BF, Zhang Y. The effect of dietary indispensable amino acid imbalances on feed intake: Is there a sensing of deficiency and neural signaling present in fish, Aquaculture 2007; 268:136-42.

31. Vásquez-Torres $W$, Pereira Filho $M$, Arias-Castellanos JA. Estudos para composiçăo de una dieta referência semipurificada para avaliçăo de exigências nutricionais em juvenis de pirapitinga, Piaractus brachypomus (Cuvier, 1818). Rev
Brasil Zootecn. 2002; 31:283-92.

32. Pezzato LE, Barrios MM, Pezzato AC, Miranda EC, Quintero PLG, Furuya WM. Relación energía: proteína en la nutrición de alevinos de piauçu (Leporinus macrocephalus). Rev Med Vet y Zoot. 2000; 47:2-7.

33. Van der Meer MB, Faber $R$, Zamora JE, Verdegem MCJ. Effect of feeding level on feed losses and feed utilization of soya and fish meal diets in Colossoma macropomum (cuvier), Aquacult Res. 1997; 28:391-403.

34. Morillo M, Visbal T, Rial L, Ovalles F, Aguirre P, Medina AL. Alimentación de alevines de Colossoma macropomum con dietas a base de Erythrina edulis y soya. Interciencia 2013; 38:1-7. 